\title{
A Revolution in Health Care Sector Through Data Sciences
}

\author{
Rajaram $^{1}$, Amit Kumar Pandey², Sanjay Saproo ${ }^{3}$, Sanjeev Bansal ${ }^{4}$ \\ ${ }^{1}$ Research Scholar, Amity Business School, Amity University Noida, ${ }^{2}$ Associate Professor, Amity Business School, \\ Amity University Noida, ${ }^{3}$ CEO, Hamilton Medical AG, Switzerland, ${ }^{4}$ Dean, FMS, Director, Amity Business School, \\ Amity University, Noida
}

\begin{abstract}
Healthcare industry is undergoing a digital transformation. There is lot of work which is happening that leverages the newer technologies in the healthcare industry. Technological advancements will be driver for change for future ready health care industry. Health care industry, now understands the role of digital technology platforms and has started embracing it to be future ready.

Digital transformation in health care industry shall be another milestone in patient care and hospital management. Changing mind-set of care givers and customers will be a challenge for all players to think differently. Emergence of disruptive technology in health care industry has potential scope to provide error free services worldwide. This is a qualitative study in which challenges and opportunities of Technology in Health Care Industry will cover.
\end{abstract}

Keywords: Health Care industry, digital transform, artificial intelligence and disruptive technology.

\section{Introduction}

Healthcare industry is undergoing a silent transformation using the newer technologies such as AI, deep learning, big data etc. The work which has been done so far has been very encouraging and is all set to change the paradigm of the healthcare industry. Today's patients are understanding the importance of health care and they are budgeting for the same. For future persecutions such expenses shall be increase accordingly ${ }^{1}$.

The old rules will be rewritten and the complexion of the industry will change completely with newer players entering and dominating the field. Healthcare industry and its various components such as pharmaceuticals, hospitals and medical devices etc.All are set to

\section{Corresponding Author:}

Dr. Amit Kumar Pandey

Associate Professor, Amity Business School, Amity

University Noida

Mob. No.: 9811471775

e-mail: akpandey7@amity.edu undergo major transformation in their business models. Emergence of the new technology platform like Artificial intelligence, big data are used by many industries and initial trends are helping them to define their pros and cons. Another aspect of health care is to provide a safest service to their customer's transparency and disclosure with the help of technology is playing crucial role ${ }^{2}$.

Use of these emerging technologies to run business is not new and many industries have had initial success. For instance, ecommerce industry uses AI extensively to do predictive analytics and based on users' search pattern and past history, suggests various options for buyers to consider. Similarly, many other industries are using AI and machine learning ${ }^{3}$ to improve their productivity.

Technology in healthcare-will incumbents rule the new world or will there be new companies: With respect to the healthcare industry, there are many applications which are under development. Healthcare industry can greatly benefit for newer technologies as it will make the healthcare delivery more affordable, accessible and reduce medication errors. Even Artificial intelligence will help medical fraternity ${ }^{4}$ in getting second opinion(Dr Amit Kumar Pandey, 2018). Here 
in this study, researchers will reveal various aspects of medical treatment pattern on the basis of big data and artificial intelligence.

All the major pharmaceutical companies are leveraging this and have established separate departments to uncover the potential. Novartis, Pfizer and several other major pharma companies are working on big data and artificial intelligence are have demonstrated that these new technologies are helpful. Digital therapeutics is another area of scope where software based solutions treat and support different kind of diseases. Digital health care delivery system is the next frontier of curing chronic patients. It has been defined ${ }^{5}$ that (Sophie Park MPH., 2019) within the pharmaceutical industry there are many areas which are undergoing fundamental transformation. Drug discovery is being transformed with the use of new age technology. Bioinformatics, clinical trials are some of the key areas where maximum impact is being felt. Clinical trials have started using AI based apps and other features for cohort selection (patient selection) and patient monitoring. Big data is helping in identifying newer patterns in clinical trials and making it more predictive of the real world outcome.

Technology companies such as Google and Microsoft are providing platforms for various healthcare companies, so that they can build applications and improve healthcare outcomes. Microsoft is partnering with lot of companies in the healthcare segment and helping them improve their business processes and services. On one hand they are helping research based companies to understand disease patterns and make discovery more predictive while on the other hand, they also partnering with distribution and pharmacies to improve their supply chain and improve last mile connectivity for medicine delivery.

Microsoft has many services running on its cloud platform, Azure which is being used by various healthcare companies. Microsoft Genomics service, on Azure, enablesscientists, data scientists and clinicians use cloud-based genomic processing services to manage the data-rich workloads. They have partnered with St. Jude Children's Research Hospital to understand, treat and cure childhood cancer and other life-threatening diseases.

In India, Microsoft has partnered with Apollo hospitals to create an AI focused network for improving cardiovascular outcomes. Microsoft and Apollo have established National Clinical Coordination Committee (NCCC) to develop Cardiovascular Disease Risk Score using AI models. This committee consists of leading doctors from top notch medical institutes.

Google is another company which is betting big on healthcare. They are using their AI expertise and supporting healthcare companies in creating a new dimension for detection, diagnosis and treatment of diseases. Google is using its computer vision and predictive analytics to predict cardiovascular risk using patients eye scan. Normally, eye scan is used to check the signs for diabetic retinopathy. However,with advanced predictive analytics Google's platform can not only predict the5-year risk of patient developing retinopathy, it can also predict the cardiovascular risk. Based on past data, it has developed algorithm which have identified the relation between retinopathy and Cardio Vascular disease and based on that it is able to predict.

These are just some of the examples and there is much deeper research being done now. Technology companies are primarily working as enablers and are set to transform the industry. It was a long awaited transformation and it will take more time to transform entire industry. Moreover, adaptation by the society shall be another hurdle, because changes is not a matter of overnight.

Radiology is another area which is being transformed by artificial intelligence. Due to AI's power in reading and analyzing visual images, radiology is an area which will undergo ${ }^{6}$ significant disruption. Researchers at Stanford University have explored a model in which system driven diagnosis is used. To error is human and another study conducted on medical error (PAndey, 2018) where study concluded with the manmade error unintentionally. Medical care givers are always in precautionary mood to tackle the medical issues, but error take place anonymously. Burdened care givers may do mistakes while medical procedures but in the case of AI based services, rectifications of such issues are very easy. Work load of care givers reduce and chance of error is almost zero.

Challenges before health care industry: Future of technology in the healthcare looks bright, but it is not without its own sets of challenges. There are multiple challenges in the adoption of technology. Firstly, all the applications are still to be validated in real world settings. Secondly, healthcare is regulated industry guarded 
heavily by the regulators. Any change in the existing treatment or diagnostic patterns will need lot of data and real world evidence before regulators make any change. Additional challenge will be from incumbents, as many of them will fear job losses for instance radiologists, pathologists and many general physicians.

Though there are multiple challenges, however this will also create newer jobs for data scientists and other allied areas

Measuring impact: Though it is certain that emerging technologies will impact the healthcare ecosystem, however we will have to access the impact and understand, if we are using technology ${ }^{7}$ only because it is available or are we using it because it really makes an impact. The impact has to be measured on the core elements of healthcare delivery. Healthcare delivery has several players ranging from hospitals to physicians to pharmaceutical companies to pharmacy chain to diagnostics. If we have to make an impact, then we must create value which transcends all the elements and eventually improves the life of the patient. Patient is central and is the core theme for the healthcare industry to exist. Hence the key questions are, how do you measure the impact of technology ${ }^{8}$ on healthcare? Alternatively, what is the value it generates for patients?

\section{Conclusion}

Data science and newer technologies are changing the healthcare industry and are becoming an integral part. Use of these technologies and tools will improve the healthcare continuum and will make the care more effective and predictive9. Collaboration between pharmaceutical companies and technology companies will become the new normal and winners will be the players who are effectively able to blend the two disciplines.

While new technologies are likely to change the narrative of the healthcare industry but the key outcome should be measured by the impact it brings to patient's life. Any new technology should enhance patients experience and must be measured by the impact it creates on patient's quality of life.

A technology based framework should be developed by all stakeholders on which impact for each technology should be measured. This framework should be the guiding principal ${ }^{\mathbf{1 0}}$. Some of the key elements of this framework are: does it improve the clinical outcome, does it make the medicine more effective, does it help in reducing the healthcare cost etc.?

\section{References}

1. Amit Kumar Pandey, Sanjeev Bansal Sanjay Saproo, Management of patient criticality at the hospitals: Astudy in reference to second opinion, Medico-Legal Update, 2017, Vol.17, issue 1, 2428.

2. Pandey Amit Kumar, managing transparency and disclosure to prevent medical error in Indian hospitals, Indian Journal of forensic medicine and toxicology, June 2018, vol-12, issue 1, Jan-. 16-22

3. Amit Kumar Pandey, Sanjeev Bansal Sanjay Saproo, Management of patient criticality at the hospitals: A study in reference to second opinion, Medico-Legal Update, 2017, Vol.17, issue 1, 2428.

4. Amit Kumar Pandey, Sanjeev Bansal Sanjay Saproo, Management of patient criticality at the hospitals: A study in reference to second opinion, Medico-Legal Update, 2017, Vol.17, issue 1, 2428.

5. Sophie Park MPH., s. V. From treatment to prevention: An exploration, Buyer2019 vol-1, issue-1, 27-31.

6. Peter Lee, Microsoft and Apollo Hospitals introduce AI powered Cardiovascular Disease risk score, August 17, 2018 | Microsoft News Center India, https://news.microsoft.com/en-in/microsoftand-apollo-hospitals-introduce-ai-poweredcardiovascular-disease-risk-score/

7. Microsoft for Healthcare: technology and collaboration for better experiences, insights and care, https://www.the-digital-insurer.com/dia/ microsoft-healthcare

8. How Google Plans to Use AI to Reinvent The $\$ 3$ Trillion US Healthcare Industry, July 2019, https:// www.cbinsights.com/research/report/googlestrategy-healthcare/

9. How can Artificial Intelligence Help Health Care? Luke A. Renner, April 17, 2018, https:// towardsdatascience.com/how-can-artificialintelligence-help-healthcare-1a2bd72ca94b

10. Harnessing data science and Artificial intelligence in health care from policy to practices, Giles Colclough Grail Dorling FarhadRiah 2018, https:// www.wish.org.qa/wp-content/uploads/2018/11/ IMPJ6078-WISH-2018-Data-Science-181015.pdf 